\title{
ALPACA air shower array to explore 100TeV gamma-ray sky in Bolivia
}

\author{
C. Calle, ${ }^{a}$ K. Hibino, ${ }^{b}$ N. Hotta, ${ }^{c}$ Y. Katayose,${ }^{d}$ C. Kato,${ }^{e}$ S. Kato,${ }^{f}$ K. Kawata,${ }^{f}$ \\ W. Kihara, ${ }^{e}$ Y. Ko, ${ }^{e}$ H. Kojima, ${ }^{g}$ R. Mayta, ${ }^{h}$ P. Miranda, ${ }^{a}$ H. Mitsui, ${ }^{i}$ K. Munakata, ${ }^{e}$ \\ H. Nakada, ${ }^{i}$ Y. Nakamura, ${ }^{j}$ M. Nishizawa, ${ }^{k}$ S. Ogio, ${ }^{h}$ M. Ohnishi, ${ }^{f}$ T. Ohura, ${ }^{i}$
} A. Oshima, ${ }^{l}$ M. Raljevich, ${ }^{a}$ H. Rivera, ${ }^{a}$ T. Saito, ${ }^{m}$ T. Sako ${ }^{*},{ }^{f}$ T. K. Sako,,${ }^{f}$ Y. Sengoku, ${ }^{i}$ S. Shibata, ${ }^{l}$ A. Shiomi, ${ }^{n}$ M. Subieta, ${ }^{a}{ }$ N. Tajima, ${ }^{o}$ W. Takano, ${ }^{b}$ M. Takita,${ }^{f}$ Y. Tameda, ${ }^{p}$ K. Tanaka, ${ }^{q}$ R. Ticona, ${ }^{a}$ H. Tsuchiya,${ }^{r}$ Y. Tsunesada, ${ }^{h}$ S. Udo, ${ }^{b}$ K. Yagisawa, ${ }^{i}$ Y. Yokoe ${ }^{f}$

${ }^{a}$ Instituto de Investigaciones Físicas, Universidad Mayor de San Andres, La Paz, La Paz 8635, Bolivia

${ }^{b}$ Faculty of Engineering, Kanagawa University, Yokohama, Kanagawa 221-8686, Japan

${ }^{c}$ Utsunomiya University, Utsunomiya, Tochigi 321-8505, Japan

${ }^{d}$ Faculty of Engineering, Yokohama National University, Yokohama, Kanagawa 240-8501, Japan

${ }^{e}$ Department of Physics, Shinshu University, Matsumoto, Nagano 390-8621, Japan

${ }^{f}$ Institute for Cosmic Ray Research, University of Tokyo, Kashiwa, Chiba 277-8582, Japan

${ }^{g}$ Faculty of Engineering, Aichi Institute of Technology, Toyota, Aichi 470-0392, Japan

${ }^{h}$ Graduate School of Science, Osaka City University, Osaka, Osaka 558-8585, Japan

${ }^{i}$ Graduate School of Engineering Science, Yokohama National University, Yokohama, Kanagawa 240-8501, Japan

${ }^{j}$ Institute of High Energy Physics, Chinese Academy of Sciences, Shijingshan, Beijing 100049, China

${ }^{k}$ National Institute of Informatics, Chiyoda, Tokyo 101-8430, Japan

${ }^{l}$ College of Engineering, Chubu University, Kasugai, Aichi 487-8501, Japan

${ }^{m}$ Tokyo Metropolitan College of Industrial Technology, Arakawa, Tokyo 116-8523, Japan

${ }^{n}$ College of Industrial Technology, Nihon University, Narashino, Chiba 275-8576, Japan

${ }^{o}$ RIKEN, Wako, Saitama 351-0198, Japan

${ }^{p}$ Faculty of Engineering, Osaka Electro-Communication University, Neyagawa, Osaka 572-8530, Japan

${ }^{q}$ Graduate School of Information Sciences, Hiroshima City University, Hiroshima, Hiroshima 731-3194, Japan

${ }^{r}$ Japan Atomic Energy Agency, Tokai-mura, Ibaraki 319-1195, Japan

E-mail: sakodicrr.u-tokvo.ac.jp 
Andes Large area PArticle detector for Cosmic ray physics and Astronomy (ALPACA) is a new air shower array project as a collaboration between Bolivia and Japan to explore the $100 \mathrm{TeV}$ gamma-ray sky in the southern hemisphere. In a plateau near the Chacaltaya mountain at $4,740 \mathrm{~m}$ altitude, a surface detector array covering $82,800 \mathrm{~m}^{2}$ with underground water Cherenkov muon detectors of total $5,400 \mathrm{~m}^{2}$ area will be constructed. Because of $2 \mathrm{~m}$ soil overburden, the muon detectors can detect muons of $>1.2 \mathrm{GeV}$ in air showers with a high purity. Using the conventional surface array to determine the primary energy and the arrival direction, the underground muon detectors improve the gamma/hadron separation and also mass identification of primary cosmic rays. For gamma-ray showers within zenith angle of 45 degrees, ALPACA has a full effective area above $20 \mathrm{TeV}$. At $20 \mathrm{TeV}$ and $100 \mathrm{TeV}, 99 \%$ and $99.9 \%$ hadron showers are rejected, respectively, while keeping the gamma-ray detection efficiency above $90 \%$. Many interesting galactic objects can be observed with 0.2 degree angular resolution at $100 \mathrm{TeV}$ with $>2,000$ hours/year exposure. ALPACA enables us the first sensitive survey of the southern gamma-ray sky at $100 \mathrm{TeV}$ energy range that is crucial to identify $\mathrm{PeV}$ accelerating objects. Preparation for infrastructure and construction of a pathfinder array ALPAQUITA are ongoing. Scientific targets, expected performance of ALPACA including the prospects for some CR observations and current status are described.

36th International Cosmic Ray Conference -ICRC2019-

July 24th - August 1st, 2019

Madison, WI, U.S.A.

* Speaker. 


\section{Introduction}

To identify the PeV accelerators in our Galaxy, called PeVatrons, detection of $100 \mathrm{TeV}$ gammarays is crucial. Very recently the first detection of $100 \mathrm{TeV}$ gamma-rays from the Crab nebula is reported by the Tibet AS $\gamma$ collaboration [四] followed by the HAWC collaboration []. Some more $100 \mathrm{TeV}$ observations are reported in this conference. The success owes to the realization of the air shower arrays at high altitudes and the excellent separation powers between electromagnetic and hadronic showers, which enable long exposure time with high signal-to-noise ratio.

Because high altitude air shower arrays sensitive to $100 \mathrm{TeV}$ gamma rays are in operation only in the Northern hemisphere, a new array is desired in the Southern hemisphere. Andes Large area PArticle detector for Cosmic ray physics and Astronomy (ALPACA) is proposed to search the PeVatrons in the Southern hemisphere using the well established technique by the Tibet AS $\gamma$ collaboration. According to the H.E.S.S. galactic plane survey around $1 \mathrm{TeV}$ [B]], the Galactic plane around the Galactic center is known to be rich in the high energy objects. ALPACA is expected to perform a systematic study of the various types of PeVatron candidates first time in the Southern hemisphere.

In this paper, the design and the performance of ALPACA are described in Sec. $\square$ and Sec.[1]. Currently a pathfinder array ALPAQUITA, meaning 'mini ALPACA', covering the 25\% of ALPACA area is under construction. The design and status of ALPAQUITA are described in Sec.9 and the paper is summarized in Sec.5.

\section{Design of ALPACA}

The ALPACA air shower array is proposed to be constructed in a plateau at $4,740 \mathrm{~m}$ altitude near the Chacaltaya mountain in Bolivia. The latitude of the site is $16^{\circ} 23^{\prime} \mathrm{S}$ from where the Galactic center (declination is $-28.9^{\circ}$ ) can be observed with the minimum zenith angle of $12.5^{\circ}$. ALPACA is composed of a traditional array of the 401 scintillation counters in a $15 \mathrm{~m}$ interval grid covering $82,800 \mathrm{~m}^{2}$ and the water Cherenkov muon detectors with $2 \mathrm{~m}$ soil overburden covering $5,400 \mathrm{~m}^{2}$. The surface array can determine the energy and arrival direction and the muon detectors count the number of muons with the energy threshold of $1.2 \mathrm{GeV}$. The alignment of the surface array and the muon detectors are shown in Fig.W (Left). Each scintillation counter is composed of $1 \mathrm{~m}^{2} \times 5 \mathrm{~cm}$ thick plastic scintillator and two PMTs. A lead plate of $5 \mathrm{~mm}$ thick is placed above the scintillator to convert photons in the air shower into electrons and positrons. Fig.W (Right) shows the cross section view of a muon detector. The muon detectors are powerful to eliminate hadronic shower events in the gamma-ray search and also to study the mass composition of the primary cosmic rays. The technique of the muon detectors is established by the Tibet AS $\gamma$ collaboration [䧃] [5]. The specifications of ALPACA are summarized in Tab.W.

\section{Expected performance}

The detector response to the primary cosmic rays and gamma rays through air showers was studied using CORSIKA v76400 [焑] and the GEANT4 v4.10.04.p02 [四] toolkit. 
Table 1: Specifications of ALPACA

$\begin{array}{ll}\text { Location } & \text { Chacaltaya plateau } \\ \text { Longitude } & 68^{\circ} 08^{\prime} \mathrm{W} \\ \text { Latitude } & 16^{\circ} 23^{\prime} \mathrm{S} \\ \text { Altitude } & 4,740 \mathrm{~m} \text { a.s.l. } \\ \text { Surface area } & 82,800 \mathrm{~m}^{2} \\ \text { Underground muon detector area } & 5,400 \mathrm{~m}^{2} \\ \text { Number of surface detector } & 401\left(1 \mathrm{~m}^{2} \times 5 \mathrm{~cm}^{t} \text { each }\right)\end{array}$
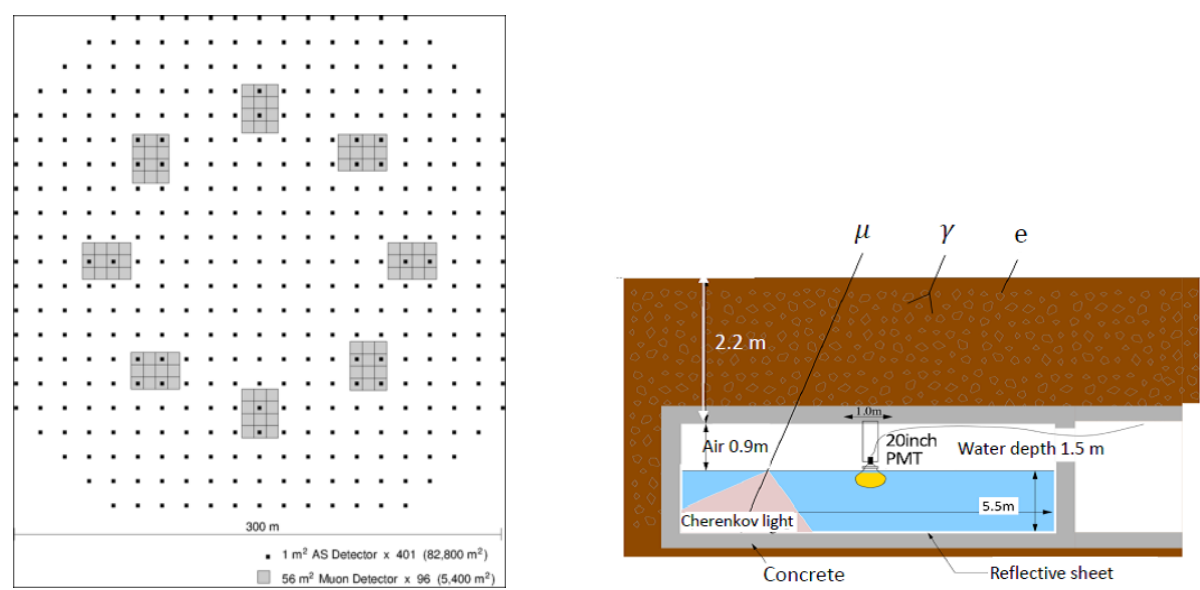

Figure 1: (Left) Array design of ALPACA. Small dots indicate the $1 \mathrm{~m}^{2}$ plastic scintillator detectors on the ground. Gray hatched squares indicate the location of the underground muon detectors. (Right) Schematic view of an underground muon detector (unit:mm).

\subsection{Energy coverage}

After applying a realistic trigger condition and quality cuts in the offline analysis, for gammaray primaries $50 \%$ and almost $100 \%$ detection efficiencies are achieved at $10 \mathrm{TeV}$ and $20 \mathrm{TeV}$, respectively. Because the detector performance rapidly changes at zenith angle, $\theta$, above $45^{\circ}$, here the results at $\theta<45^{\circ}$ are presented unless noted. For charged cosmic rays, assuming the observed spectrum and mass composition, the mode energy is at $10 \mathrm{TeV}$.

\subsection{Annual exposure}

The annual exposure time (hours) of the celestial objects visible from the ALPACA site as a function of declination is shown in Fig.D. The solid and dotted curves indicate the exposures assuming $\theta<45^{\circ}$ and $\theta<60^{\circ}$, respectively. In this calculation, a loss factor of the projection area for the inclined incidents, $\cos (\theta)$, is considered along the trajectory of each object. Some important $\mathrm{TeV}$ gamma-ray sources found in the TeVCat catalog [ [ 8$]$ is indicated according to their declinations. Including the Galactic center, for many interesting objects more than 2000 hours 


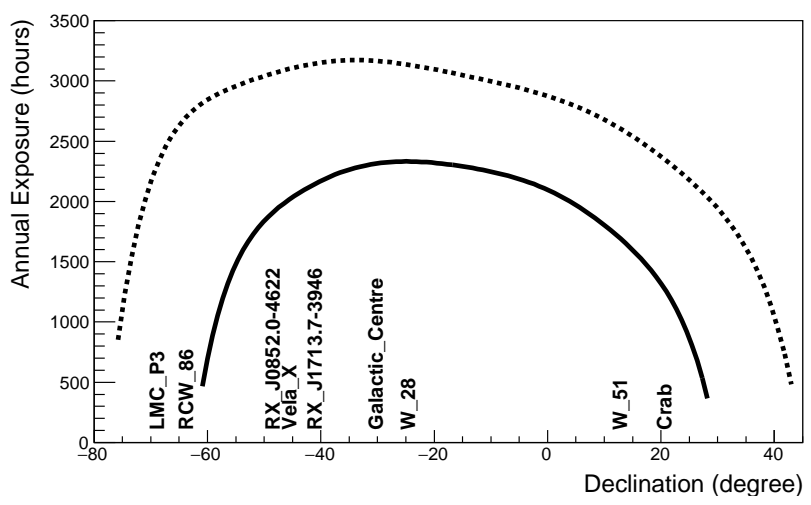

Figure 2: Annual exposure of ALPACA as a function of declination. Thick solid (dotted) line assumes zenith angle coverage up to $45^{\circ}\left(60^{\circ}\right)$. Names of major TeV gamma-ray sources are indicated at their declinations.

exposure is possible even with $\theta<45^{\circ}$. Interestingly more than 1000 hours exposure is available for Crab so that the cross calibration with the Northern experiments is possible.

As discussed in Sec.1B.], above $45^{\circ}$ the threshold energy significantly increases. At $45^{\circ}<$ $\theta<60^{\circ}$ the full efficiency is achieved at $200 \mathrm{TeV}$. This is still an interesting energy and the the gain in exposure time and declination coverage is significant while it is noted that the analysis of such inclined shower events is a new challenge.

\subsection{Gamma/hadron separation}

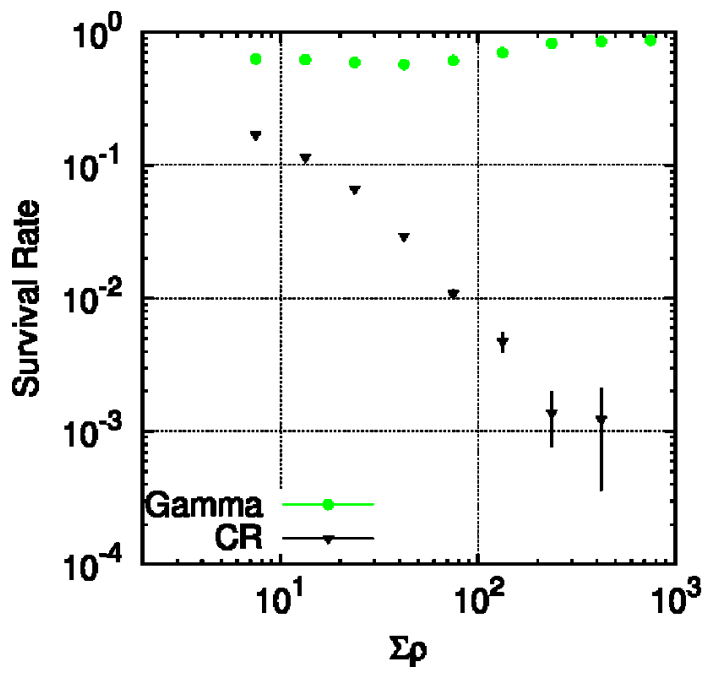

Figure 3: Selection efficiencies for gamma (circle) and proton (triangle) primary showers as a function of the shower size $(\Sigma \rho)$ after applying event selection using the signal of the muon detector. Gamma-ray showers with $\Sigma \rho=40$ and 400 correspond to the primary energies of $10 \mathrm{TeV}$ and $100 \mathrm{TeV}$, respectively.

In each event the number of muons is measured using the muon detectors. Comparing the events with same shower size, the gamma-ray initiated showers have less muons than the cosmic- 
ray initiated hadronic showers. Applying a shower size dependent event selection using the number of muons, most of the hadron shower background events are eliminated while keeping a high gamma-ray survival efficiency. Fig.[] shows the survival efficiencies of gamma-ray events and cosmic-ray events as a function of the shower size, $\Sigma \rho$, after applying an event selection. Here $\Sigma \rho$ is defined as a sum of number of particles recorded in the scintillation counters. The shower size $\Sigma \rho=40$ and 400 correspond to the primary gamma-ray energies of approximately $10 \mathrm{TeV}$ and $100 \mathrm{TeV}$. From Fig. 1$]$ background events are eliminated to $0.1 \%$ at $100 \mathrm{TeV}$ while $>90 \%$ gammaray events survive.

\subsection{Sensitivity}

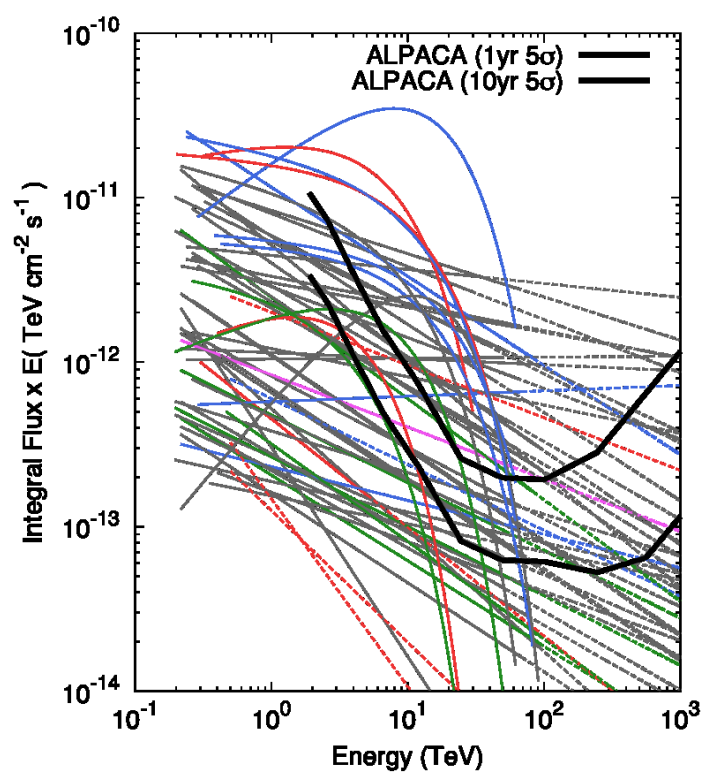

Figure 4: Five sigma sensitivities of ALPACA for 1 year and 10 year observations. Spectra of the H.E.S.S. sources visible in the ALPACA field of view are shown together. Extrapolations from the H.E.S.S. detection are expressed with dashed lines.

Based on the performance discussed above, the $5 \sigma$ sensitivity of ALPACA is shown in Fig.t. Two thick curves are sensitivities after 1 and 10 year operations. Various thin curves indicate the energy spectra reported in the H.E.S.S. galactic plane survey [3]]. The solid curves are based on the H.E.S.S. detection while the dashed curves are extrapolations from the lower energy detections. It is found that H.E.S.S. detected strong spectral cutoff between $10 \mathrm{TeV}$ and $100 \mathrm{TeV}$ from many sources and ALPACA can confirm the maximum energies of these accelerators. The H.E.S.S. catalog also contains a lot of objects with hard spectral indexes without apparent cutoff up to multi $10 \mathrm{TeV}$. These are the prime candidates of PeVatrons well in the sensitivity of ALPACA.

\subsection{Cosmic-ray observations}

Anisotropy in the arrival direction of charged cosmic rays is a hot topic in this field [Q]. So far only IceCube/IceTop experiment covers the southern sky from the South pole. ALPACA at 
the moderate latitude can fulfill the southern coverage with sufficient overlaps with the northern experiments and the IceCube/IceTop.

It is known that the number of muons in air showers is sensitive to the mass of the primary cosmic ray. The muon detectors of ALPACA open a new possibility of the mass composition study using its large area and high muon purity. Recently ultra-high-energy observations report a lack of muon numbers in the hadronic air shower simulations [ए]]. It is noted that the energy coverage of ALPACA overlaps with the direct measurements by the space-borne experiments and also with the collision energies realized in the operational hadron colliders, RHIC and LHC. Comparing with these measurements at the same energy range, ALPACA can place a solid anchor point in the muon problem.

\section{Pathfinder array ALPAQUITA}

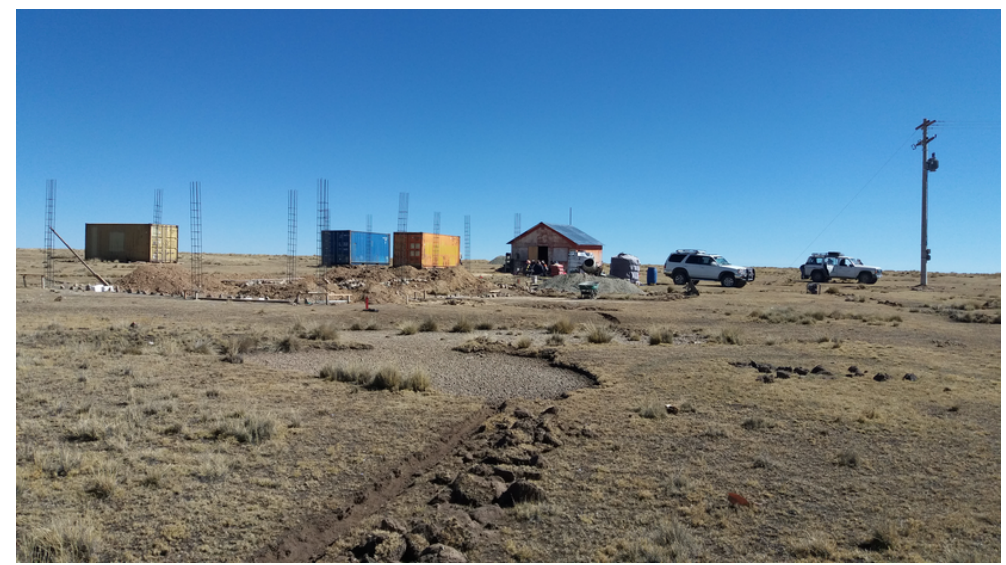

Figure 5: A photo of the ALPACA site in the middle of June 2019. Three containers at the left side contain the scintillation counters and data acquisition system for ALPAQUITA, those are installed in the new hut under construction in front of them. The new power line is seen in the right side. The hut in the middle is for workers.

Currently ALPACA is only partly funded and the pathfinder array covering $25 \%$ of the full ALPACA area, called ALPAQUITA, is under construction. The surface array with 97 scintillation counters and the muon detectors of $1000 \mathrm{~m}^{2}$ coverage are constructed in 2019 and 2020, respectively. Before the array construction, preparation for infrastructure is on going as shown in Fig.[5. A new power line, a hut for data taking and maintenance works, guard fence, will be ready by August 2019 , then the deployment of the surface array starts. Water will be supplied from a well about $1 \mathrm{~km}$ away from the site. The water supply system will be ready by 2020 .

\section{Summary}

The technique of $100 \mathrm{TeV}$ gamma-ray detection is established in the Northern hemisphere. Based on the established technique, ALPACA aims to search $100 \mathrm{TeV}$ gamma-ray sources with a sufficient sensitivity first time in the Southern hemisphere. The pathfinder array ALPAQUITA will 
be operational in 2019 with the surface array and in 2020 with the muon detectors. ALPAQUITA will detect several bright sources expected by the H.E.S.S. galactic plane survey. Once the full ALPACA is complete, different categories of $\mathrm{TeV}$ gamma-ray sources are systematically studied for their abilities of $\mathrm{PeV}$ acceleration. The unique location in latitude and the muon detectors of ALPACA allow new possibilities of studying charged cosmic rays and their high energy hadronic interactions.

\section{Acknowlegments}

ALPACA is supported by the Japan Society for the Promotion of Science (JSPS) through Grantsin-Aid for Scientific Research (A) 19H00678, Scientific Research (B) 19H01922, and by the joint research program of the Institute for Cosmic Ray Research (ICRR), The University of Tokyo. KK is supported by the Toray Science Foundation.

\section{References}

[1] The Tibet AS $\gamma$ Collaboration, Phys. Rev. Lett., in press (2019).

[2] The HAWC Collaboration, arXiv:1905.12518 (2019).

[3] The HESS Collaboration, A\&A 612, A1 (2018).

[4] T. K. Sako, K. Kawata, M. Ohnishi, A. Shiomi, M. Takita, H. Tsuchiya, Astropart. Phys. 32, 177-184 (2009).

[5] The Tibet AS $\gamma$ Collaboration, ApJ, 813:98 (2015).

[6] D. Heck, J. Knapp, J. N. Capdevielle, G. Schatz, T. Thouw, Report FZKA, 6019, Forschungszentrum Karlsruhe (1998).

[7] S. Agostinelli et al., Nucl. Instrum. Meth. Phys. Res. A 506, 250 (2003).

[8] http://tevcat.uchicago.edu

[9] The Tibet AS $\gamma$ Collaboration, ApJ, 836:153 (2017).

[10] H. P. Dembinski, et al., EPJ Web of Conferences 210, 02004 (2019). 